\title{
Miracle Mouthwash Plus Hydrocortisone
}

National Cancer Institute

\section{Source}

National Cancer Institute. Miracle Mouthwash Plus Hydrocortisone. NCI Thesaurus.

Code C118293.

An oral rinse containing miracle mouthwash and the corticosteroid hydrocortisone, with potential anti-mucositic activity. Upon rinsing with this mouthwash, the ingredients in the miracle mouthwash may provide analgesia; the hydrocortisone modulates the production of pro-inflammatory cytokines which may prevent inflammation of the oral mucosa, and reduce chemotherapy- and/or radiation-induced oral mucositis. 